\title{
Numerical Simulation of Particle Motion in an Ultrasound Field Using the Lattice Boltzmann Model
}

\author{
J. A. Cosgrove ${ }^{\dagger}$, J. M. Buick ${ }^{\ddagger}$, D. M. Campbell ${ }^{\dagger}$ and C. A. Greated ${ }^{\dagger}$ \\ $\nmid$ †epartment of Physics and Astronomy The University of Edinburgh \\ The Kings Buildings, Mayfield Road, Edinburgh EH9 3JZ, Scotland UK. \\ $\ddagger$ Physics and Electronics, School of Biological, Biomedical and Molecular Sciences \\ University of New England, Armidale, NSW 2351, Australia
}

\begin{abstract}
In this paper we investigate the motion of small particles suspended in a fluid through which an ultrasound field is propagating. The application of the lattice Boltzmann model to this problem is considered using a two dimensional model. Particles in an ultrasound field are observed to move with a mean particle motion. Further, the time-averaged force on a fixed cylinder is computed and found to be in good agreement with a theoretical expression for the radiation force. Simulations are performed with a single particle, although the approach can equally be applied for a larger number of particles.
\end{abstract}

PACS: 07.05.Tp; 43.25.Mn; 43.25.Qp

Keywords: Simulation; micron particles; radiation pressure; lattice Boltzmann model.

\section{Introduction}

When an ultrasound beam propagates through a liquid it will interact with any particles which are suspended in the fluid. In general all fluids will contain small particles of some description. Tap water is contaminated with a variety of small particles, some of which can be removed, for 
example using a fine filter; however, a number of smaller particles will remain. The interaction of particles with ultrasound waves is relevant to many medical applications, for example noninvasive imaging, physiotherapy, Doppler ultrasound and lithotripsy. Particles can also be added, for example contrast agents can be used to increase the resolution of an ultrasound scan [1] and seeding particles are added when performing a fluid measurement using an optical technique such as particle image velocimetry [2]. It is therefore useful to have a method for determining the effect that the ultrasound and the particle have on each other. This can be a complex interaction, for example, an impedance mismatch between the particle and the fluid with produce a reflection of the acoustic wave which will impose a radiation force on the particle and alter the ultrasound field. Other effects such as acoustic streaming around the particle will further modify the behaviour. In this paper we introduce a numerical scheme based on the lattice Boltzmann model and assess its ability and potential for simulating the interaction between a fluid, an ultrasound field and a small particle.

\section{The Lattice Boltzmann Model}

\section{Fluid Model}

The lattice Boltzmann model, see for example [3, 4], is a relatively new technique which has been utilised in a number of fluid mechanics simulations and more recently its application has been extended to problems in acoustics. There are a number of different forms in which the model can be applied. Attention here is restricted to the specific model used in the current approach. The technique is applied on a regular square grid in which each grid point is connected to its four horizontal and vertical neighbours by links $\boldsymbol{e}_{1}, \ldots \boldsymbol{e}_{4}$, and to its four diagonal neighbours by links $\boldsymbol{e}_{5}, \ldots \boldsymbol{e}_{8}$. These are shown in Figure 1 and are give by $\boldsymbol{e}_{1}=(1,0), \boldsymbol{e}_{2}=(0,1), \boldsymbol{e}_{3}=(-1,0)$, 
$\boldsymbol{e}_{4}=(0,-1), \boldsymbol{e}_{5}=(1,1), \boldsymbol{e}_{6}=(-1,1), \boldsymbol{e}_{7}=(-1,-1)$ and $\boldsymbol{e}_{8}=(1,-1)$. Distribution functions, $f_{i}, i=1, \ldots, 8$, which describe the motion of the fluid in the direction of $\boldsymbol{e}_{i}$ are then introduced, as is the distribution function $f_{0}$ and the null link vector $\boldsymbol{e}_{0}=(0,0)$. These distribution functions, at position $\boldsymbol{x}_{s}$ and time $t_{s}$, evolve according to the Boltzmann equation:

$$
f_{i}\left(\boldsymbol{x}_{s}+\boldsymbol{e}_{i}, t_{s}+1\right)-f_{i}\left(\boldsymbol{x}_{s}, t_{s}\right)=-\frac{1}{\tau}\left[f_{i}\left(\boldsymbol{x}_{s}, t_{s}\right)-\bar{f}_{i}\left(\boldsymbol{x}_{s}, t_{s}\right)\right]
$$

where

$$
\bar{f}_{i}=\rho_{s} w_{i}\left[1+3 \boldsymbol{e}_{i} \cdot \boldsymbol{u}_{s}+\frac{9}{2}\left(\boldsymbol{e}_{i} \cdot \boldsymbol{u}_{s}\right)^{2}-\frac{3}{2} u_{s}^{2}\right]
$$

with $w_{0}=4 / 9, w_{1}=w_{2}=w_{3}=w_{4}=1 / 9$, and $w_{5}=w_{6}=w_{7}=w_{8}=1 / 36$ [5]. It can be shown that this scheme satisfies the Navier Stokes equation [3] and simulates a fluid with kinematic viscosity $\nu_{s}=2(\tau-1) / 6$, density $\rho_{s}=\sum_{i=0}^{8} f_{i}$ and velocity $\boldsymbol{u}_{s}=\left(\sum_{i=0}^{8} f_{i} \boldsymbol{e}_{i}\right) / \rho_{s}$. Here $\tau$ is a free parameter which can be selected to give the desired value of $\nu_{s}$.

\section{Acoustic Model}

Acoustic waves can be introduced into this model provided the pressure variations remain small relative to the ambient pressure $[6,7,8]$. A wide range of linear and non-linear waves fall into this range with pressure variations of less than $1 \%$. The lattice Boltzmann model applied here has

the speed of sound $c_{s}=1 / \sqrt{3}$; the pressure is found from the equation of state: $p_{s} / \rho_{s}=c_{s}^{2}$. In the simulations presented here the ultrasound field is introduced at the grid boundaries parallel to $\boldsymbol{e}_{2}$. This is done by setting the values of $f_{i}$ at these boundary points to the values of $\bar{f}_{i}\left(\rho_{s}^{A}(t), \boldsymbol{u}_{s}^{A}(t)\right)$, where $\rho_{s}^{A}(t)$ and $\boldsymbol{u}_{s}^{A}(t)$ are the acoustic density and velocity at the boundary. A square grid was used with an integer number of wavelengths in each direction. The values of the acoustic density and velocity are therefore given by $\rho_{s}^{A}(t)=\rho_{s}^{(0)}+\delta \rho_{s} \sin \left(\omega_{s} t_{s}\right)$ and $\boldsymbol{u}_{s}^{A}(t)=\boldsymbol{\delta} \boldsymbol{u}_{s} \sin \left(\omega_{s} t_{s}\right)$ for a 
travelling wave and $\rho_{s}^{A}(t)=\rho_{s}^{(0)}$ and $\boldsymbol{u}_{s}^{A}(t)=\boldsymbol{\delta} \boldsymbol{u}_{s} \sin \left(\omega_{s} t_{s}\right)$ for a standing wave, where $k_{s}$ and $\omega_{s}$ are the wave number and angular frequency of the ultrasound wave and $\rho_{s}^{(0)}$ is the ambient density; $\delta \rho_{s}$ and $\delta \boldsymbol{u}_{s}=\left(\delta \rho_{s} c_{s} / \rho_{s}^{(0)}, 0\right)$ are the amplitudes of the density and velocity variations respectively. The values used for these parameters will be discussed later. Periodic boundary conditions were applied at the other grid boundaries.

\section{Particle Model}

In this application we consider a solid particle, following the approach of Ladd [9], further described in [10]. Initially the particle, whose size spans several lattice sites, is placed on the computational grid. Fluid is allowed both outside and inside the particle. This means that the grid points inside the particle can be treated in the same manner as those outside. This is done for computational convenience and there is no direct interaction between the fluid inside and outside the particle. The fluid inside the particle relaxes quickly to travel with the same velocity as the particle. This simulates a rigid particle. The perimeter of the particle cuts a number of grid links and this determines the boundary between the fluid inside and outside the particle. Boundary conditions are applied at this perimeter such that momentum is transferred between the particle and the exterior fluid. This is a two-way process; the fluid momentum acts to change the particle momentum and vice-versa. The change in the fluid momentum occurs directly through the distribution functions at the boundary. The momentum change at the boundary is then calculated. This momentum change is then equated to a force acting on the particle which alters its velocity. At each time step the new position of the centre of mass of the particle is calculated and the particle is moved accordingly. The position of the particle is rounded to the centre of the links between nodes. In principle it is also possible to calculate the torque imposed on the particle and hence the change in its angular momentum. Here we consider only one particle in a plane 
wave in which case the net torque will be zero.

\section{Units}

In the simulations the unit of length is the grid spacing, the unit of time is the time step and the unit of density is derived from the density of the fluid, for example, when we state $c_{s}=1 / \sqrt{3}$, we mean that the speed of sound is equal to $1 / \sqrt{3}$ grid spacings per time step. To perform meaningful simulations these quantities must be related to the real quantities in SI units. This is done using dimensionless quantities and an example is shown in Appendix A. Throughout this paper the subscript $s$ is used for physical quantities from the simulations, measured in lattice units; a subscript $r$ refers to the real world value and the appropriate SI unit is given. The subscripts are dropped for equations which can apply to either system of units.

\section{Radiation Pressure}

Wu et al. [11] present an analytical theory for the radiation force on a rigid cylinder in a standing wave field when the diameter is much smaller than the wavelength. In this case the radiation force per unit length on cylinder is [11]

$$
F=B S V \omega \frac{(\delta p)^{2}}{\rho^{(0)} c^{3}}
$$

where $S=\sin (2 k h), V$ is the volume of a cylinder per unit length, $h$ is the position of the centre of the cylinder along the $x$-axis, $\delta p$ is the acoustic pressure at the center of the cylinder as it would be if the cylinder were absent, and ${ }^{1} B=\left\{[(1-\beta) /(1+\beta) / 2+1 / 4\}\right.$, where $\beta=\rho^{(0)} / \rho^{(1)}$, and $\rho^{(1)}$ is the density of the cylinder. Equation (3) is compared with experiment [11] for frequencies of 2.29 and $1.01 \mathrm{MHz}$. A glass microneedle of radius $40 \mu \mathrm{m}$ was used as the cylinder. The

\footnotetext{
${ }^{1}$ Correcting a typo in the final line of equation (24) in [11].
} 
displacement of the cylinder was measured at different acoustic pressures and related to the force on the microneedle by a calibration procedure. Good agreement was found between theory and experiment.

\section{Results}

The simulations were run on a $N$ by $N$ grid, with $N \geq 10 r_{s}$, where $r_{s}$ is the particle radius. In each case the acoustic field was evolved for four periods at which time the particle was introduced into the fluid. This was done to ensure that the initial conditions did not effect the simulation. Figure 2 shows a typical particle trajectory, normalised by the amplitude of the particle displacement $a_{r}$ over the length of the simulation. After a few periods the particle motion is seen to be a superposition of a sinusoidal motion and a steady procession. The steady procession is indicated by two parallel lines drawn through the maxima and minima of the particle displacement. There is also a time lag between the acoustic wave and the particle motion. This is highlighted by the insert in Figure 2 showing a magnified view of the displacement wave. The position of the particle is such that the fluid displacement (in the absence of a particle) is maximum at $t=n T$, where $n$ is an integer. Here we see that the particle maximum occurs slightly after $16 T$. A similar time lag was found at all displacement maxima and was measured to be approximately $0.03 T$. This corresponds is a small time lag between a change in the fluid velocity and a corresponding change in the particle velocity. This is to be expected given the finite mass of the particle. In a wave period the distance which the particle advances is small compared to its amplitude of oscillation, $a_{r}$, which is itself small; however over a large number of periods the displacement can be significant. 
The results presented in Figure 2 and described above indicate the general features of the particle motion. To investigate the details it is necessary to consider a realistic example. Here we consider a $10 \mathrm{MHz}$ acoustic wave with intensity $185 \mathrm{Wcm}^{-2}$ and particles with radii ranging from $5-10 \mu \mathrm{m}$. The propagation medium is a body fluid with viscosity $\mu_{r}=\nu_{r} \rho_{r}^{(0)}=4.0$ mPas and density $\rho_{r}^{(0)}=1,000 \mathrm{Kgm}^{-3}$, where the speed of sound is $c_{r}=1,500 \mathrm{~ms}^{-1}$. Figure 3 shows the procession velocity $v_{r}$ as a function of radius. In each case a progressive velocity, $v_{r}$ of between 0.7 and $1.0 \mathrm{mms}^{-1}$ was measured and $v_{r}$ generally seems to increase with the particle radius. In Figure 4 the velocity and pressure profile in the vicinity of the particle for $r_{r}=6 \mu \mathrm{m}$ is shown. The figure shows the distortion of the velocity and pressure field due to the presence of the particle. These effects occur on a spatial scale similar to the radius of particle, however, it is easily resolved with the spatial resolution of the LBM simulation; the resolution has been greatly reduced in Figure 4 for clarity.

Since the particles are close to the ultrasound source it is likely that the streaming velocity will be small and the radiation force will be significant, even for such a small particle. This is supported by the increase in the procession velocity with particle radius (see Figure 3) which would not occur if the particles were simply moving with the streaming velocity. Further, when the simulation was run without a particle present, no streaming motion was observed. To investigate whither the mean motion is produced by the radiation pressure the process is compared with equation (3). Here it is necessary to consider the time-averaged force acting on a fixed cylinder. For a moving particle the radiation pressure will be canceled by the viscous force and the time-averaged force is zero. Measurements of the radiation force were obtained using a standing wave and a cylinder positioned such that $S=1$. Half-way boundary conditions were applied. This means that a fluid particle which is travelling from a fluid node into a cylinder 
node is reflected half-way between the two nodes and returns to the fluid node in a single timestep with its momentum reversed. This gives a boundary which is half-way between the fluid and the cylinder nodes with $\beta=1$. The rate of change of momentum due to the reflections is then calculated from which the force on the cylinder is determined [12]. The force measurements were obtained by considering the rate of change of the fluid momentum at the sites. Figure 5 shows the measured radiation pressure as a function of the particle radius for a $10 \mathrm{MHz}$ standing wave with Mach number 0.0035 in the same fluid. Also shown is the analytical expression [11]: equation (3). Good agreement is observed between the LBM results and theory for radii upto $40 \mu \mathrm{m}$ indicating that the motion of the particles is due to the radiation pressure. The theory [11] assumes that the radius us the cylinder is small with respect to the wavelength. A $10 \mathrm{MHz}$ wave has a wavelength of $150 \mu \mathrm{m}$ which accounts for the increased differences at larger radii. Another possible source of error is the formation of acoustic streaming. To minimise this the cylinder was placed close to the source (the grid boundary). When the simulation was performed without a cylinder, no streaming motion was observed during the period of measurement. It is, however, possible that streaming motion in the boundary layer of the cylinder may impose a small influence on the results. It is also evident that the object represents a cylinder with rough edges due to the discrete nature of the lattice. This can be accounted for by considering an effective radius. Koch and Ladd [12] determine that the effective radius is larger than the actual radius by 0.3 of a lattice unit. This is considered to be negligible here since the radius is always greater then $10 \mathrm{lu}$. 


\section{Conclusion and Further Work}

We have demonstrated the ability of the LBM to investigate the motion of suspended particles in an ultrasound field. The oscillating motion of the particle was modelled, as was a procession motion due to the radiation stress. The velocity and pressure fields in the vicinity of the particle were also measured. The time-averaged force on a fixed, rigid cylinder in an acoustic field was also computed and compared with a theoretical expression for the radiation force. A good agreement was found indicating that the motion of the particles was induced by the radiation pressure. Possibilities for extending this work are extending the model to three dimensions, including more particles and using non-rigid particles to better simulate contrast agents by considering either particles with compliant boundaries [13] or immiscible fluid 'particles' [14].

\section{Appendix A}

Dimensionless quantities must be used to convert between the lattice units of the simulation and SI units which are used to interpret the results. An example of how this is done is presented in this Appendix where, as in the rest of the paper, a subscript $s$ is applied to quantities in lattice Boltzmann units and the subscript $r$ to real world values. Consider a drift velocity of $v_{s}=3.4$ $\times 10^{-7}$ measured from a simulation with $\tau=0.53, \rho_{s}^{(0)}=1$ and $\lambda_{s}=1,000$, where the particle has radius $r_{s}=67$ which represents a wave propagating through a body fluid with viscosity $\mu_{r}$ $=\nu_{r} \rho_{r}^{(0)}=4.0 \mathrm{mPas}$ and density $\rho_{r}^{(0)}=1,000 \mathrm{Kgm}^{-3}$, where the speed of sound is $c_{r}=1,500$ $\mathrm{ms}^{-1}$. Then the acoustical Reynolds number defined as $R e=c \lambda / \nu$ gives:

$$
R e_{s}=\frac{1 / \sqrt{3} \times 1000}{(2 \times 0.53-1) / 6}=R e_{r}=\frac{1500 \lambda_{r}}{4 \times 10^{-6}} \text {. }
$$


Thus $\lambda_{r}=0.15 \mathrm{~mm}$, corresponding to a frequency of $f_{r}=10 \mathrm{MHz}$. Now, comparing the wave period and the wavelength we see that 1732 time-steps $\Longleftrightarrow 100$ ns and 1000 lattice spaces $\Longleftrightarrow 0.15$ $\mathrm{mm}$, so $r_{r}=10 \mu \mathrm{m}$. Finally the measured velocity in the simulations (both the particle velocity and the drift velocity) can be converted to physical units using the above relationships which is equivalent to calculating their ratio with respect to the speed of sound. Thus, $v_{s} / c_{s}=v_{r} / c_{r}$, so $v_{r}=0.9 \mathrm{mms}^{-1}$. The intensity of the ultrasound beam can be found from $\delta u_{r}^{A}$, calculated as above, using the formula: $I=u^{2} \rho^{(0)} c / 2[15]$.

Equation (3) can be re-written in dimensionless form as

$$
\frac{F}{\omega V \rho^{(0)} c}=B S M^{2}
$$

where $M$ is the Mach number of the flow. This enables the force measured in the simulation to be related to the force on a real particle.

\section{References}

[1] F. Calliada, R. Campani, O. Bottinelli, A. Bozzini, and M. G. Sommaruga. Ultrasound contrast agents. Basic principles. European Journal of Radiology, 27:S157-S160, 1998.

[2] J. A. Cosgrove, J. M. Buick, S. D. Pye, and C. A. Greated. PIV applied to Eckart streaming produced by a medical ultrasound transducer. Ultrasonics, 39:461-464, 2001.

[3] S. Chen and G. D. Doolen. Lattice-Boltzmann method for fluid flows. Annual Review of Fluid Mechanics, 30:329-364, 1998. 
[4] D. A. Wolf-Gladrow. Lattice-gas cellular automata and lattice Boltzmann models. In Lecture Notes in Mathematics 1725, editor, A. Dold and F. Takens and B. Teissier. Springer, 2000.

[5] Y. H. Qian, D. d'Humières, and P. Lallemand. Lattice BGK models for Navier-Stokes equation. Europhysics Letters, 17:479-484, 1992.

[6] J. M. Buick, C. A. Greated, and D. M. Campbell. Lattice BGK simulation of sound waves. Europhysics Letters, 43:235-240, 1998.

[7] J. M. Buick, C. L. Buckley, C. A. Greated, and J. Gilbert. Lattice Boltzmann BGK simulation of non-linear sound waves: The development of a shock front. Journal of Physics A: Mathematical and General, 33:3917-3928, 2000.

[8] D. Haydock and J. M. Yeomans. Lattice Boltzmann simulations of acoustic streaming. Journal of Physics A: Mathematical and General, 34:5201-5213, 2001.

[9] A. J. C. Ladd. Numerical simulations of particulate suspensions via a discretized Boltzmannequation. 1. Theoretical foundation. Journal of Fluid Mechanics, 271:285-309, 1994.

[10] A. J. C. Ladd and R. Verberg. Lattice-Boltzmann simulations of particle-fluid suspensions. Journal of Statistical Physics, 104:1191-1251, 2001.

[11] J. Wu, G. Du, S. S. Work, and D. M. Warshaw. Acoustic radiation pressure on a rigid cylinder: An analytical theory and experiments. J. Acoust. Soc. Am., 87 (2):581-586, 1990.

[12] D. L. Koch and A. J. C. Ladd. Moderate Reynolds number flows through periodic and random arrays of aligned cylinders. Journal of Fluid Mechanics, 349:31-66, 1997. 
[13] Z. Lin H. Fang and Z. Wang. Lattice Boltzmann simulation of viscous fluid systems with elastic boundaries. Physical Review E, 57:R25-R28, 1998.

[14] E. Orlandini, M. R. Swift, and J. M. Yeomans. A lattice Boltzmann model of binary-fluid mixtures. Europhysics Letters, 32 (6):463-468, 1995.

[15] L. E. Kinsler, A. R. Frey, A. B. Coppens, and J. V. Sanders. Fundamentals of Acoustics Third Edition. John Wiley and Sons, New York, 1982.

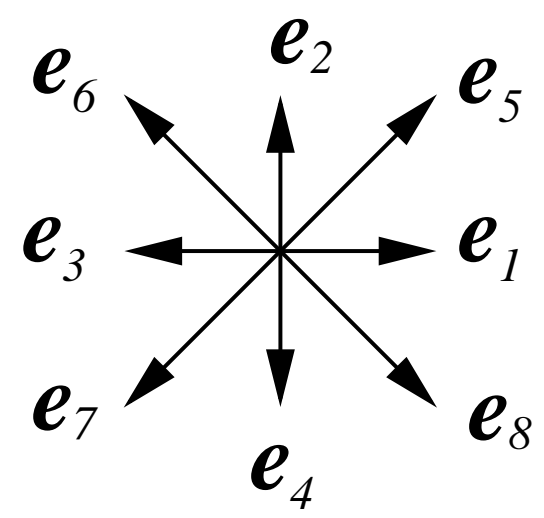

Figure 1: Numerical grid and definition of the link vectors 


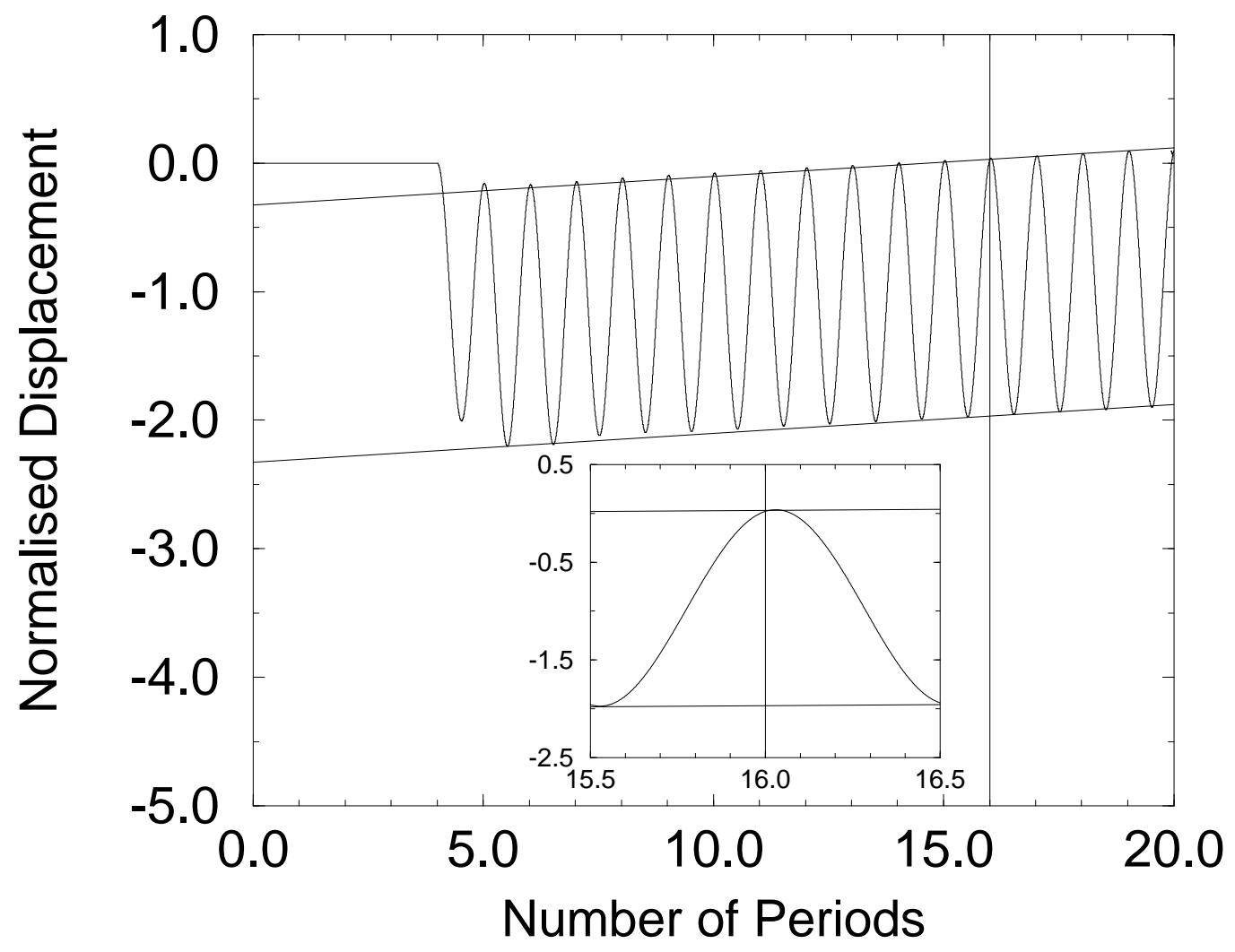

Figure 2: A typical graph of particle displacement 


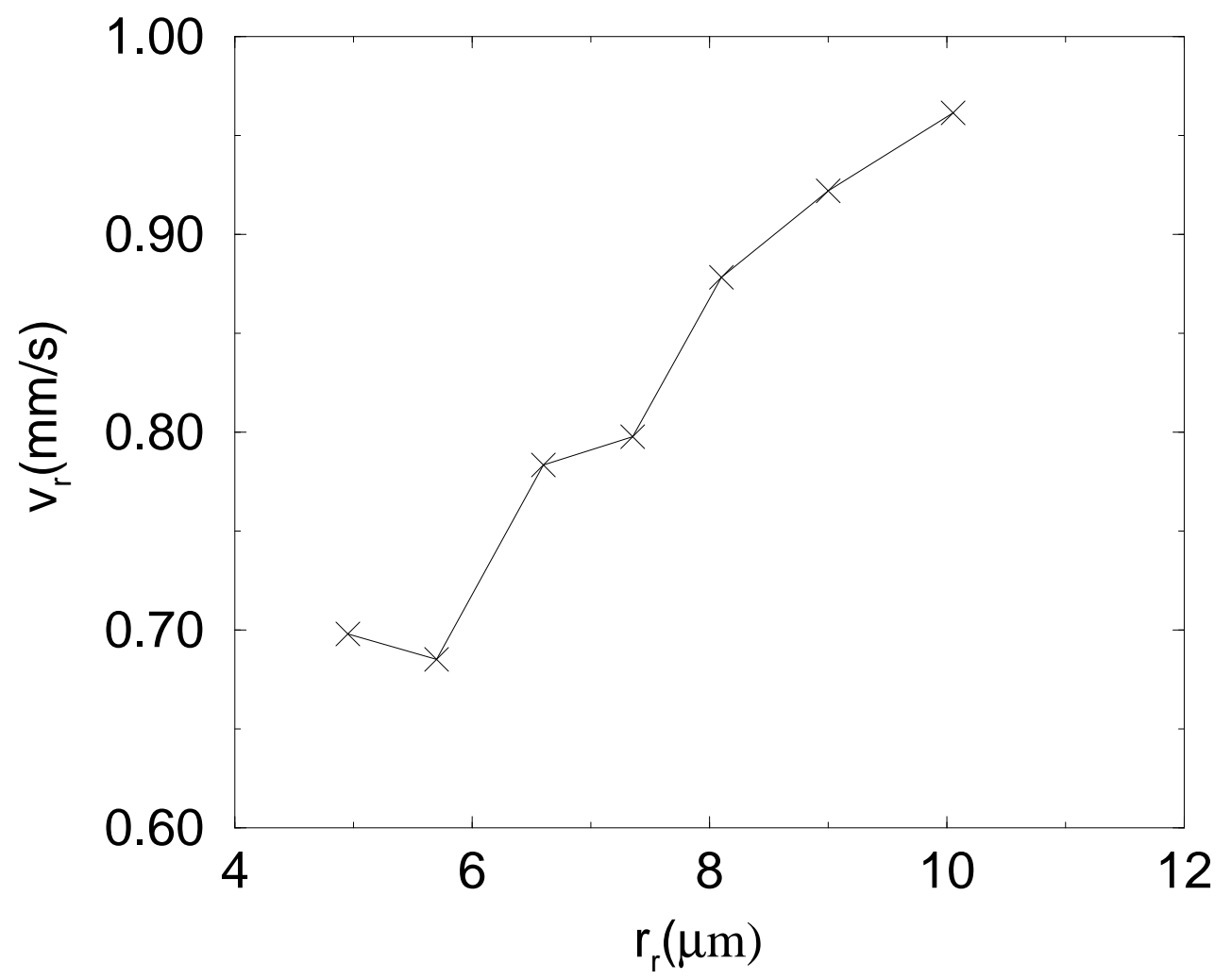

Figure 3: The progressive velocity $v_{r}$ as a function of particle radius $r_{r}$ for a $10 \mathrm{MHz}$ wave with an intensity $I_{r}=185 \mathrm{Wcm}^{-2}$. 


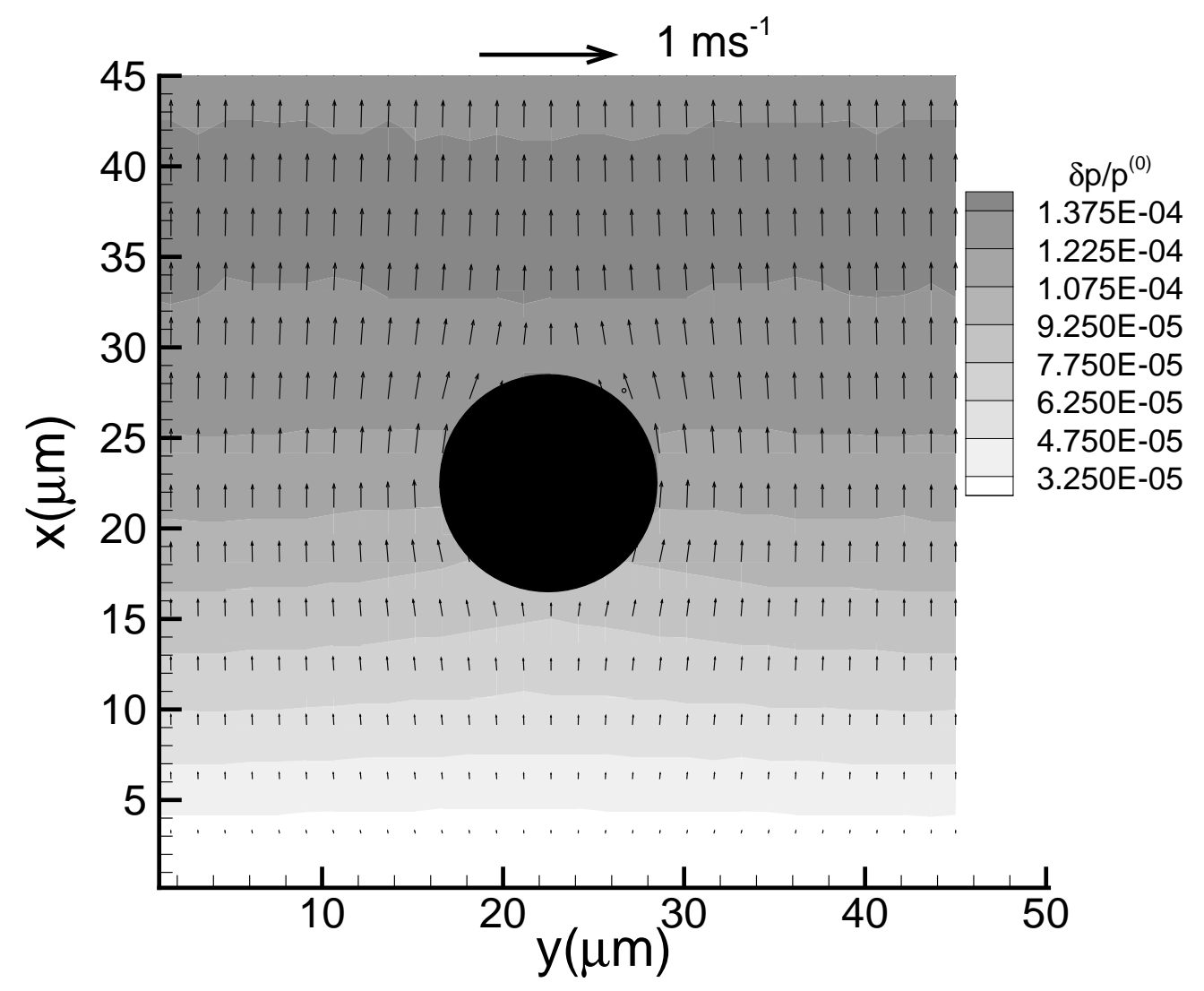

Figure 4: The velocity and pressure field in the vicinity of a solid particle. The particle has $r_{r}=$ $6 \mu \mathrm{m}$ and the ultrasonic field has frequency $10 \mathrm{MHz}$ and intensity $183 \mathrm{Wcm}^{-2}$. 


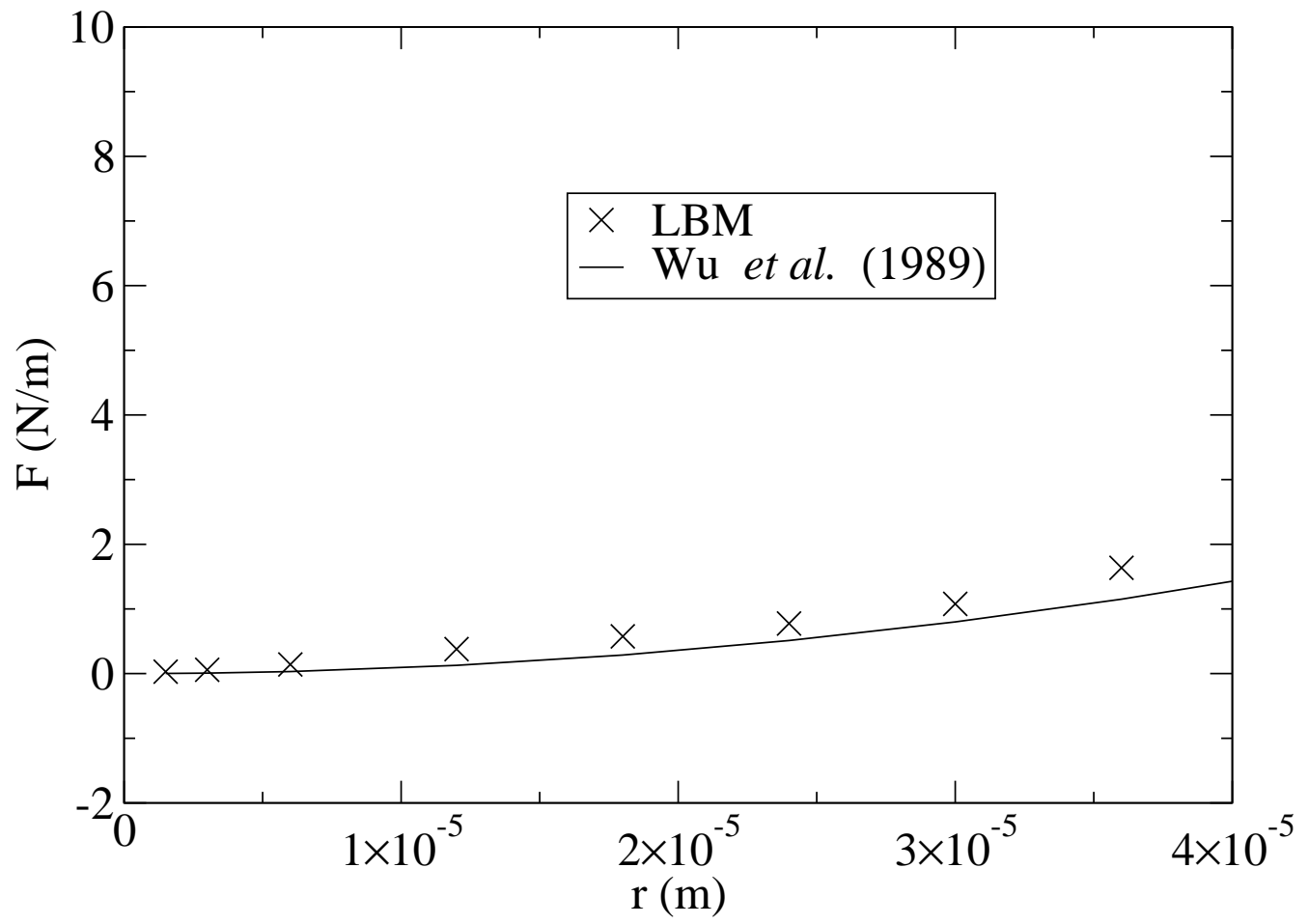

Figure 5: The radiation force per unit length on a cylinders of varying radii calculated using the LBM for a $10 \mathrm{MHz}$ standing wave with $M=0.0035$. Equation (3) is also shown for comparison. 